Modern Physics Letters A,

(C) World Scientific Publishing Company

\title{
A NO-GO THEOREM FOR DE SITTER COMPACTIFICATIONS?
}

\author{
N.D. HARI DASS. \\ Chennai 600113 , INDIA \\ Received (received date) \\ Revised (revised date)
}

Institute of Mathematical Sciences, C.I.T Campus,

\begin{abstract}
A general framework for studying compactifications in supergravity and string theories was introduced by Candelas, Horowitz, Strominger and Witten 4 . This was further generalised to take into account the warp factor by de Wit, Smit and Hari Dass 6 .Though the prime focus of the latter was to find solutions with nontrivial warp factors (shown not to exist under a variety of circumstances), it was shown there that de Sitter compactifications are generically disfavoured (see also 3 ). In this note we place these results in the context of a revived interest in de Sitter spacetimes .
\end{abstract}

\section{Introduction and preliminaries.}

There is renewed interest in de Sitter spacetimes both from the microscopic quantum gravity point of view $\mathbf{t}$ as well as from the macroscopic cosmological point of view $\mathrm{E}$. This interest has been triggered on the one hand by cosmological observations pointing towards an accelerating universe and on the other hand by a variety of conceptual issues with de Sitter quantum gravity. Models for explaining an accelerating universe make use of either a cosmological constant of the right sign or exotic matter(also called quintessence) which can provide negative pressure. It is also possible to invoke both. The interesting question is whether Superstring and Supergravity theories can naturally accommadate either or both of these.

A lot of progress has been made in our understanding of supergravity and superstring theories. Nevertheless, attempts to confront these developments with the known phenomenology of elementary particles have not been easy. In a pioneering work, Candelas et al 1 set up a general framework to analyse the ground state configurations of such theories which admit compactification of the higher dimensional theories into a four-dimensional Minkowski spacetime and an internal manifold usually taken to be compact. More generally they looked for compactifications of the type

$$
M^{d} \rightarrow M^{4} \times M^{d-4}
$$

where $M^{d-4}$ is a compact space and $M^{4}$ is maximally symmetric space-time (Minkowski, (anti-)de Sitter). In Candelas et al 1 this was done by taking

$$
g_{M N}(x, y)=\left(\begin{array}{cc}
g_{\mu \nu}^{0}(x) & 0 \\
0 & g_{m n}(y)
\end{array}\right)
$$


A No-go theorem for de Sitter Compactifications.... ...

However, the most general metric (in suitable choice of coordinates) 6 is of the form $(\Delta \geq 0)$

$$
g_{M N}(x, y)=\left(\begin{array}{cc}
\Delta^{-1}(y) g_{\mu \nu}^{0}(x) & 0 \\
0 & g_{m n}(y)
\end{array}\right)
$$

The corresponding vielbein (upto a tangent space rotation) is

$$
E_{M}^{N}(x, y)=\left(\begin{array}{cc}
\Delta^{-1 / 2}(y) e_{\mu}^{0 \alpha}(x) & 0 \\
0 & e_{m}^{a}(y)
\end{array}\right)
$$

We have addressed the issue of the restrictions placed on $M^{4}$ from the higher dimensional Einstein's equations on the one hand(sec.2) and from requirements of residual supersymmetry on the other(sec.3). In the case of the former what is really required is to split the higher dimensional equation into its four-dimensional counterpart as well as the $d$-4-dimensional counterpart. The technical ingredients required for this are outlined in Appendix A. An important step in this direction is a general categorisation of energy-momentum tensors; all the known supergravity theories come under this class. It is then shown that the compactness of the internal manifold imposes severe restrictions and the most important consequence is that de Sitter compactifications are ruled out. The analysis of residual supersymmetry is in itself not very restrictive but when combined with Bianchi identities they too turn out to give the same restrictions. Because of the very general nature of our considerations, it would be very hard to evade our conclusions. In sec.4 we have analysed the extent to which the compactness of $M^{d-4}$ can be relaxed.

\section{On-shell analysis.}

We start by writing down the higher-dimensional Einstein equations:

$$
\hat{R}_{M N}-\frac{1}{2} g_{M N} \hat{R}+\hat{T}_{M N}=0
$$

equivalently

$$
\hat{R}_{M N}+\hat{T}_{M N}-\frac{1}{d-2} g_{M N} \hat{T}=0
$$

In these equations $\hat{T}_{M N}$ is the higher-dimensional stress tensor and $\hat{T}$ the higherdimensional trace of it. On using the maximal symmetry of $M^{4}$ (see eqn(A.8)) one finds

$$
\hat{T}=4 t+T
$$

Decomposing the higher-dimensional Einstein equation into its 4-dimensional and 6-dimensional parts:

$$
\begin{gathered}
3 m_{4}^{2}-\frac{1}{2} \Delta D^{m}\left(\Delta^{-3} \partial_{m} \Delta\right)+\frac{((d-6) t-T)}{(d-2) \Delta}=0 \\
R_{m n}-2 \Delta^{1 / 2} D_{m}\left(\Delta^{-3 / 2} \partial_{n} \Delta\right)+T_{m n}-g_{m n} \frac{(T+4 t)}{d-2}=0
\end{gathered}
$$


We now consider a class of energy-momentum tensors that are quadratic in $p$-forms $F_{M-1 . . M_{p}}(1 \leq p \leq d-1)$ :

$$
\begin{aligned}
& \hat{T}_{M N}=\sum_{p} \hat{T}_{M N}^{(p)} \\
& \hat{T}_{M N}^{(p)}=p \hat{F}_{M Q_{1} . . Q_{p-1}} \hat{F}_{N}^{Q_{1} \ldots Q_{p-1}}-\frac{g_{M N}}{2} \hat{F}_{Q_{1} . . Q_{p}} \hat{F}^{Q_{1} . . Q_{p}}
\end{aligned}
$$

Such energy-momentum tensors occur for antisymmetric gauge fields $(\hat{F}$ is the covariant field strength) $(3 \leq p \leq d-3)$, Yang-Mills field strengths $(p=2, d-2)$, and scalar fields $(p=1, d-1)$. All the supergravity theories considered here have energy mimentum tensors of this type $\mathrm{G}$. In compactifications with a maximally symmetric 4-dimensional spacetime the nonvanishing components are

$$
\begin{aligned}
\hat{F}_{m_{1} \ldots m_{p}} & =F_{m_{1} \ldots m_{p}} \\
\hat{F}_{\mu \nu \rho \sigma m_{1} \ldots m_{p-4}} & =i e^{0} \epsilon_{\mu \nu \rho \sigma} f_{m_{1} \ldots m_{p-4}} \quad(p \geq 4)
\end{aligned}
$$

This leads to

$$
\begin{aligned}
T_{m n}^{(p)}= & p F_{m q_{1} . . q_{p-1}} F_{n}^{q_{1} . . q_{p-1}} \\
& -{ }^{p} C_{5} \Delta^{4} f_{m q_{1} . . q_{p-5}} f_{n}^{q_{1} . . q_{p-5}} \\
& -\frac{1}{2} g_{m n}\left(F^{2}-{ }^{p} C_{4} \Delta^{4} f^{2}\right) \\
t^{(p)}= & -\frac{1}{2}\left(F^{2}+{ }^{p} C_{4} \Delta^{4} f^{2}\right)
\end{aligned}
$$

so that

$$
\begin{aligned}
(d-6) t^{(p)}-T^{(p)=} & -(p-1) F^{2} \\
& -{ }^{p} C_{4}(d-p+1) \Delta^{4} f^{2}
\end{aligned}
$$

The crucial point is that this is negative for $2 \leq p \leq d-2$ and zero for $p=1, d-1$. Even though we have considered compactifications of the type $M^{d-4} \times M^{4}$ all the remarks generalise to the $M^{d-D} \times M^{D}$ cases also.

We now rewrite the four-dimensional field eqn (7) as

$$
\frac{3 m_{4}^{2}}{\Delta}-\frac{D^{m}\left(\Delta^{-3} \partial_{m} \Delta\right)}{2}+\frac{\Delta^{-2}((d-6) t-T)}{d-2}=0
$$

and integrate over the compact internal space. On using the negativity of $(d-6) t-T$ and the fact that the integral of the divergence is zero one finds

$$
m_{4}^{2} \geq 0
$$

Which means that the de Sitter case $\left(m_{4}^{2}<0\right)$ is ruled out!. If we had started with higher dimensional Einstein's equations with a cosmological constant $\Lambda$ (our conventions are : $\Lambda>0$ is de Sitter), this condition would become

$$
3 m_{4}^{2}+\frac{\Lambda}{(d-2) \Delta} \geq 0
$$


If the higher dimensional theory is Anti-de Sitter $(\Lambda<0)$, the four dimensional world can only be anti-de Sitter.

Returning to the analysis without $\Lambda$, if $M^{4}$ is (flat)Minkowski spacetime one gets further restrictions

$$
m_{4}^{2}=0 \rightarrow(d-6) t^{(p)}-T^{(p)}=0
$$

Consequently

$$
D^{m}\left(\Delta^{-3} \partial_{m} \Delta\right)=0 \rightarrow \Delta=\text { const } .
$$

The field strengths are also constrained by eqn(16):

$$
\hat{F}_{M_{1} . . M_{p}}=0 \quad 2 \leq p \leq d-2
$$

It should be noted that to arrive at eqn (14) it is not mandatory that $M^{6}$ has to be compact; it suffices for $\Delta(y)$ and the various field strengths to have appropriate asymptotic fall off conditions. This point will be elaborated later.

The derivatives of $\Delta$ do not always occur as in eqn (13). In later applications the following remark will be of importance: if $f(\Delta)$ is a positive function and $a, b$ are arbitrary constants $(b \neq 0)$, then

$$
f(\Delta)\left[a\left(\partial_{m} \Delta\right)^{2}+b \partial_{m} \partial_{m}(\ln \Delta)\right]=g(\Delta) \partial_{m} X_{m}
$$

where $g(\Delta)$ is another positive function

$$
g(\Delta)=f(\Delta) \Delta^{-a / b}
$$

and $X_{m}$ is the vector:

$$
X_{m}=b \Delta^{\frac{a-b}{b}} \partial_{m} \Delta
$$

\section{Residual Supersymmetry.}

In the previous section we analysed the Einstein's equations to address the issue of possible compactifications. One may not want to impose classical field equations for a variety of reasons. In the context of string theory, for example, the zero-slope limit may yield corrections. Of course, these higher derivative corrections are very small in the long distance limit of these theories.

Another point to keep in mind is the fact that it is desirable for compactifications to maintain some residual supersymmetry to control quantum fluctuations or solve the so called hierarchy problem.

In what follows we shall use only the On-shell supersymmetry transformations. These along with Bianchi identities are shown to imply contracted field equations though not the full ones. Nevertheless these are quite restrictive.

It is actually desirable to use Off-shell supersymmetry transformations. But offshell formulations of supersymmetry are very difficult to obtain. They are available only for type-I supergravity theories without super-Yang-Mills multiplets. 
In the next few subsections we illustrate how considerations of residual supersymmetry when used in conjunction with suitable Bianchi identities turn out to be almost as restrictive as the on-shell analysis of the previous section. Since only on-shell supersymmetry transformations are used this will amount to something in between a fully off-shell analysis and an on-shell analysis. It should be emphasised that if full supersymmetry is used as versus residual supersymmetry one would just recover the full bosonic field equations.

\section{1. $d=10, N=1$ Supergravity.}

The field content of this theory, also called $d=10, N=1$ Einstein-Maxwell supergravity, is: the zehnbein $E_{M}^{A}$, the gravitino field $\Psi_{M}$, a field strength $H_{M N P}$, a scalar field $\phi$ and a spinor field $\lambda$. In addition one has the field strengths $F_{M N}^{A}$ and spinors $\chi^{A}$ for the Yang-Mills multiplet.

The fermionic supersymmetry transformations modulo terms of higher order in the fermionic fields are:

$$
\begin{aligned}
\delta \Psi_{M} & =D_{M} \epsilon+\frac{\sqrt{2}}{32} e^{2 \phi}\left(\Gamma_{M}^{N P Q}-9 \delta_{M}^{N} \Gamma^{P Q}\right) H_{N P Q} \epsilon \\
\delta \lambda & =\left(\sqrt{2} \Gamma^{M} D_{M} \phi+\frac{1}{8} e^{2 \phi} \Gamma^{M N P} H_{M N P}\right) \epsilon \\
\delta \chi^{A} & =-\frac{1}{4} F_{M N}^{A} \Gamma_{M N} \epsilon
\end{aligned}
$$

As we will be working in a background where all fermion fields vanish, the terms higher order in the fermion fields do not matter. On using eqn (3) we can write these as

$$
\begin{aligned}
\delta \psi_{\mu} & =D_{\mu} \epsilon-i \gamma_{\mu} \gamma_{5} \Delta^{-1 / 2} T \epsilon \\
\delta \psi_{m} & =D_{m} \epsilon+\frac{\sqrt{2}}{32} e^{2 \phi}\left(\Gamma_{m} H-12 H_{m}\right) \epsilon \\
\delta \lambda & =\left(\sqrt{2} i \Gamma^{m} \partial_{m} \phi+\frac{1}{8} i e^{2 \phi} H\right) \epsilon \\
\delta \chi^{A} & =-\frac{1}{4} F_{m n}^{A} \Gamma^{m n} \epsilon
\end{aligned}
$$

where

$$
\begin{aligned}
T & =-\frac{1}{4} i \Gamma^{m} \partial_{m} \ln \Delta+\frac{i \sqrt{2}}{32} e^{2 \phi} H \\
H & =H^{m n p} \Gamma_{m n p} \quad H^{m}=H^{m n p} \Gamma_{n p}
\end{aligned}
$$

The detailed analysis of this theory can be found in 1 . Here we will only highlight the main line of reasoning. The integrability condition for $\delta \psi_{\mu}=0$ is (after use has been made of the maximal symmetry of $M^{4}$ ):

$$
\left(T^{2}+\frac{1}{4} m_{4}^{2} \Delta\right) \epsilon=0
$$


A No-go theorem for de Sitter Compactifications.... ...

Combining this with the $\delta \lambda$ equation one finds

$$
\begin{aligned}
\frac{1}{64} \sqrt{2} & \left\{\Gamma^{m} \partial_{m}\left(\phi+\frac{1}{2} \ln \Delta\right), e^{2 \phi} H\right\} \epsilon \\
= & -\frac{1}{4} \Delta\left(m_{4}^{2}-\partial_{m}\left(\Delta^{-1 / 2} e^{\phi}\right) \partial^{m}\left(\Delta^{-1 / 2} e^{-\phi}\right)\right) \epsilon
\end{aligned}
$$

The matrix multiplying $\epsilon$ on the l.h.s is antihermitian which can only have imaginary eigenvalues. Thus the real eigenvalue occurring in eqn (26) must be zero:

$$
m_{4}^{2}=\partial_{m}\left(\Delta^{-1 / 2} e^{\phi}\right) \partial^{m}\left(\Delta^{-1 / 2} e^{-\phi}\right)
$$

In a compact space every function must have a maximum unless it is constant. Therefore

$$
m_{4}^{2}=0 \rightarrow \Delta^{1 / 2} e^{\phi}=\text { const }
$$

Thus we see that in this case considerations of residual supersymmetry rule out both de Sitter and anti-de Sitter compactifications.

For the allowed Minkowski case one can draw further conclusions. After a lot of algebra (see 2 for details) one finds

$$
\begin{aligned}
& \sqrt{2} e^{2 \phi} \Gamma^{m n p q}\left(D_{m} H_{n p q}+\frac{1}{4} \sqrt{2} F_{m n}^{A} F_{p q}^{A}\right) \eta \\
= & \left(e^{2 \phi}\left(F_{m n}^{A}\right)^{2}+6 e^{4 \phi} H^{2}-16 D^{2} \phi-64\left(\partial_{m} \phi\right)^{2}\right) \eta
\end{aligned}
$$

where $\eta$ is a commuting 6 -dimensional spinor. The classical Bianchi identity for this theory is of the form:

$$
D_{m} H_{n p q}+\frac{1}{4} \sqrt{2} F_{m n}^{A} F_{p q}^{A}=0
$$

Using this in eqn (29) one gets

$$
e^{2 \phi}\left(F_{m n}^{A}\right)^{2}+6 e^{4 \phi} H^{2}-16 D^{2} \phi-64\left(\partial_{m} \phi\right)^{2}=0
$$

Using the result of eqn (19) it is easily seen that

$$
\partial_{m} \phi=0 ; \quad \partial_{m} \Delta=0 ; \quad F_{m n}^{A}=0 ; \quad H_{m n p}=0
$$

It turns out that anomaly cancellations in this theory require the classical Bianchi identity of eqn (30) to be modified to

$$
D_{[m} H_{n p q]}=-\frac{1}{4} \sqrt{2} F_{[m n}^{A} F_{p q]}^{A}+a R_{[m n}^{a b} R_{p q]}^{a b}
$$

where $a$ is a constant fixed by the anomaly-free condition. Therefore it is no longer possible to obtain eqns (31, 32). As was done in 4 , some components of the gauge fields can be identified with the spin connection

$$
F_{[m n}^{A} F_{p q]}^{A}=2 \sqrt{2} a R_{[m n}^{a b} R_{p q]}^{a b}
$$


Quite miraculously an alternate expression emerges out of the residual supersymmetry analysis:

$$
\frac{\sqrt{2} e^{2 \phi} \Gamma D H}{32} \eta=\left[\frac{3 e^{4 \phi} H^{2}}{16}-2\left(\partial_{m} \phi\right)^{2}-\frac{D^{2} \phi}{2}\right] \eta
$$

Using this and eqns $(33,34)$ one arrives at

$$
\frac{3}{16} e^{4 \phi} H_{m n p}^{2}-2\left(\partial_{m} \phi\right)^{2}-\frac{1}{2} D^{m} D_{m} \phi=0
$$

Applying our earlier techniques to a compact $M^{6}$ one again gets the same conclusions as before. Also $M^{6}$ turns out to be Ricci-flat.

\subsection{Nonchiral $d=10, N=2$ Supergravity}

This is a theory that can descend from the $d=11, N=1$ supergravity theory. Therefore we analyse the latter. The field content of this $d=11$ supergravity theory is an elfbein $E_{M}^{A}$, a gravitino field $\Psi_{M}$ and a field strength $F_{M N P Q}$. The Bianchi identity for $F_{M N P Q}$ is

$$
\partial_{[M} F_{P Q R S]}=0
$$

We leave out all the details (which can be found in a) and just state that the consequences of residual supersymmetry reduce to the condition

$$
\begin{aligned}
& -\frac{\sqrt{2}}{432}\left(\Gamma^{m p q r s} D_{m} F_{p q r s}+2 i \Delta^{2} \Gamma^{m} \partial_{m} f\right) \epsilon \\
& =\left\{m_{4}^{2} \Delta-\frac{F^{2}}{216}-\frac{2 f^{2} \Delta^{4}}{(144)^{2}}+\frac{\left(\partial_{m} \ln \Delta\right)^{2}}{3}-\frac{D^{2} \ln \Delta}{6}\right\} \epsilon
\end{aligned}
$$

Again the l.h.s is proportional to the Bianchi identity for $F_{M N P Q}$. Imposing this identity one is led to

$$
m_{4}^{2} \Delta-\frac{F_{m n p q}^{2}}{216}-\frac{2 f^{2} \Delta^{4}}{(144)^{2}}+\frac{\left(\partial_{m} \ln \Delta\right)^{2}}{3}-\frac{D^{2} \ln \Delta}{6}=0
$$

and our earlier techniques applied to a compact $M^{6}$ give the following: The de Sitter case $m_{4}^{2}<0$ is ruled out.

If one considers the flat Minkowski case $\left(m_{4}^{2}=0\right)$ which is permitted by eqn (39) one can draw further conclusions i.e

$$
m_{4}^{2}=0 \rightarrow \Delta=\text { const, } F_{m n p q}=0 .
$$

Once again the result is that the warp factor has to be trivial for the Minkowski case.

The anti-de Sitter case $\left(m_{4}^{2}>0\right)$ is also permitted by eqn (39). But in this case no further restrictions can be extracted. Both the warp factor and the field strengths $\left(F_{m n p q}, f\right)$ can be nontrivial. In fact explicit solutions of this kind are known (for references see 3 ). 


\subsection{Chiral $d=10, N=2$ Supergravity.}

This ten dimensional theory(also called IIB) does not descend from $d=11$ supergravity theory. The field content of this theory consists of a zehnbein $E_{M}^{A}$, a gravitino field $\Psi_{M}$, a spinor field $\lambda$, a complex field strength $G_{M N P}$, a self-dual field strength $F_{M N P Q R}$, a complex vector $P_{M}$ and a $U(1)$-gauge field $Q_{M}$.

The selfduality condition for the field strength is:

$$
F_{M N P Q R}=\frac{i}{120} E \epsilon_{M N P Q R S T U V W} F^{S T U V W}
$$

The Bianchi identities for both $F, G$ take the form:

$$
\begin{aligned}
\left.\partial_{[M} F_{N P Q R S}\right] & =\frac{5 i}{12} G_{[M N P} G_{Q R S]}^{*} \\
D_{[M} G_{N P Q]} & =-P_{[M} G_{N P Q]}^{*}
\end{aligned}
$$

There are Bianchi identities for $Q_{M}, P_{M}$ also which can also be viewed as MaurerCartan equations associated with the coset space $S U(1,1) / U(1)$.

$$
\begin{aligned}
\partial_{[M} Q_{N]} & =-i P_{[M}^{*} P_{N]} \\
D_{[M} P_{N]} & =0
\end{aligned}
$$

One can solve eqns (43) to eliminate $P_{M}, Q_{M}$ in terms of two real scalar fields (the dilaton and axion). Once again we leave out all the details(which can be found in 2 ) and just state the consequences of residual supersymmetry along with the Bianchi identities:

$$
3 m_{4}^{2} \Delta-\frac{D^{2} \ln \Delta}{2}-\left(\partial_{m} \ln \Delta\right)^{2}-4 f^{2}-\frac{\left|G_{m n p}\right|^{2}}{48}=0
$$

Analysing this equation for compact manifolds shows that de Sitter case is ruled out.

For the Minkowski case the additional consequences are

$$
\Delta=\text { const }, f_{m}=0, G_{m n p}=0 .
$$

Further use of the Maurer-Cartan equations also yields $P_{M}=0$ and Ricci-flatness.

For the anti-de Sitter case no such restrictions are implied by eqn (44).

\section{Asymptotic fall off in noncompact $M^{6}$}

The analysis presented so far has treated $M^{6}$ as a compact space. But with suitable asymptotic fall-off conditions the same conclusions can be reached even for noncompact $M^{6}$. The volume of $M^{6}$ is $\int d^{6} y \sqrt{\operatorname{det} g_{m n}}$. When this volume diverges we have the noncompact case. When $M^{6}$ is noncompact it is necessary that the integral $\int d^{6} y \sqrt{\operatorname{det} g_{m n}} \Delta^{-1}(y)$ exists for an effective four dimensional description to exist. If we introduce spherical coordinates on $M^{6}$ and take spatial infinity to be at $Y \rightarrow \infty$ where $Y$ is the radial coordinate, it is clear that $\Delta^{-1}$ must fall off at least 
$\simeq Y^{-6}$. A consistency check on this is provided by the divergence term in eqn (13). This leads to a surface integral of the type $\int d S_{m} \Delta^{-3} \partial_{m} \Delta$ and this should vanish asymptotically.The fall-off of $\Delta^{-1}$ is indeed compatible with this. Now it follows that from eqn (13) that $t, T$ must fall of faster than $\Delta$ which is no problem at all. Under these fall-off conditions the conclusions previously reached on the basis of on-shell analysis for the compact case hold in the noncompact case as well.

The generalisation of the analysis based on residual symmetry to the noncompact case works differently for the three cases considered in sec.3. For the type-I case the condition in eqn (27) was obtained without recourse to any compactness. In the noncompact case this equation can have nontrivial solutions.

In order to extend the analysis of the nonchiral case of sec3.2 we use eqns (19,20,21) to cast eqn (39) as

$$
m_{4}^{2} \Delta-\frac{F_{m n p q}^{2}}{216}-\frac{2 f^{2} \Delta^{4}}{(144)^{2}}-\frac{\Delta^{2}}{6} D_{m} \Delta^{-3} \partial_{m} \Delta=0
$$

Dividing the whole equation by $\Delta^{2}$ we find that extension to the noncompact case is exactly along the same lines as that of the on-shell analysis.

Finally to extend the analysis of sec.3.3 to the noncompact case, note that by dividing eqn (44) by $\Delta^{2}$ one again verifies that the terms involving the derivatives of $\Delta$ fall off sufficiently fast and the conclusions reached for the compact case hold again.

\section{Conclusions.}

One sees that our analysis disfavours de Sitter compactifications in a variety of circumstances. Furthermore, when $M^{4}$ is flat-Minkowski, it also shows that the warp factor has to be trivial. The original analysis assumed that $M^{6}$ was compact but sec.4 of this note shows how the analysis can be extended to the noncompact case. We had to be content with only the on-shell supersymmetry transformations. It is desirable to find off-shell extensions.

The present analysis should be extended to cosmological solutions where only the 3 -geometry is taken to be maximally symmetric. There are indications that the solution space can be much larger in that case $\mathbf{6}$. In addition to the issue of the cosmological constant one also has to address the issue of exotic energy momentum tensors i.e quintessence $\mathbf{b}$. Whether quintessence can occur naturally

in these theories is still an outstanding issue $\mathrm{E}$. The implications of our results for models like the Randall-Sundrum model 10 should be investigated.

\section{Acknowledgments}

I would like to thank Naresh Dadhich and Dharam Ahluwalia for organising a very stimulating meeting. 


\section{Appendix A}

In this appendix we give various formulae that are important for the analysis carried out in the main text. The anholonomity (Ricci rotation) coefficients for the vielbien of eqn(3) are given by

$$
\Omega_{A B}^{C}=2 E_{A}^{M} E_{B}^{N} \partial_{[M} E_{N]}^{C}
$$

The spin-connection in terms of $\Omega_{A B}^{C}$ is given by :

$$
\omega_{M}^{A B}=\frac{1}{2} E_{M}^{C}\left(\Omega_{A B}^{C}-\Omega_{B C}^{A}-\Omega_{C A}^{B}\right)
$$

Finally, the higher-dimensional curvature tensor, denoted by $\hat{R}$ is given by:

$$
\hat{R}_{M N}^{A B}=\partial_{M} \omega_{N}^{A B}-\partial_{N} \omega_{M}^{A B}-2 \omega_{[M}^{A C} \omega_{N]}^{C B}
$$

It's nonvanishing components are (for details see 1 ):

$$
\begin{aligned}
\hat{R}_{\mu \nu}^{\alpha \beta} & =R_{\mu \nu}^{\alpha \beta}+\frac{1}{2} e_{\mu}^{[0 \alpha} e_{\nu}^{0 \beta]} \Delta^{-3} g^{m n} \partial_{m} \Delta \partial_{n} \Delta \\
\hat{R}_{\mu n}^{\alpha b} & =-\frac{1}{2} e_{\mu}^{0 \alpha} D_{n}\left(\Delta^{-3 / 2} e_{b}^{m} \partial_{m} \Delta\right) \\
\hat{R}_{m n}^{a b} & =R_{m n}^{a b}=\partial_{m} \omega_{n}^{a b}-\partial_{n} \omega_{m}^{a b}-2 \omega_{[m}^{a c} \omega_{n]}^{c b}
\end{aligned}
$$

The maximal symmetry of $M^{4}$ implies 6

$$
\begin{aligned}
R_{\mu \nu}^{\alpha \beta} & =\partial_{\mu} \omega_{\nu}^{\alpha \beta}-\partial_{\nu} \omega_{\mu}^{\alpha \beta}-2 \omega_{[\mu}^{\alpha \gamma} \omega_{\nu]}^{\gamma \beta} \\
& =2 m_{4}^{2} e_{[\mu}^{0 \alpha} e_{\nu]}^{0 \beta}
\end{aligned}
$$

We shall also be needing the higher-dimensional Ricci tensor:

$$
\hat{R}_{M N}=\hat{R}_{M P}^{A B} E_{N A} E_{B}^{P}
$$

The nonvanishing components of this Ricci-tensor are:

$$
\begin{aligned}
\hat{R}_{\mu \nu} & =R_{\mu \nu}-\frac{1}{2} g_{\mu \nu}^{0} \Delta D^{m}\left(\Delta^{-3} \partial_{m} \Delta\right) \\
& =g_{\mu \nu}^{0}\left\{3 m_{4}^{2}-\frac{1}{2} \Delta D^{m}\left(\Delta^{-3} \partial_{m} \Delta\right)\right\} \\
\hat{R}_{m n} & =R_{m n}-2 \Delta^{1 / 2} D_{m}\left(\Delta^{-3 / 2} \partial_{n} \Delta\right)
\end{aligned}
$$

Maximal symmetry of $M^{4}$ implies that the components of the d-dimensional energymomentum tensor $\hat{T}_{M N}$ have the form:

$$
\begin{aligned}
\hat{T}_{\mu \nu} & =g_{\mu \nu} t=\Delta^{-1} g_{\mu \nu}^{0} t \\
\hat{T}_{m n} & =T_{m n} \quad T=g^{m n} T_{m n}
\end{aligned}
$$




\section{References}

[1] P. Candelas, G.T. Horowitz, A. Strominger and E. Witten, Nucl. Phys. B258 (1985) 46.

[2] B. de Wit, D.J. Smit and N.D. Hari Dass, Nucl. Phys. B283 (1987) 165.

[3] G.W. Gibbons, Aspects of Supergravity theories in Supersymmetry Supergravity and Related topics eds. F. del Aguila, J.A. Azcarraga and L.E. Ibanez (World Scientific, 1985) pp346-351; J. Maldacena and C. Nunez, Int.. Jour. Mod. PhysA16(2001)822.

[4] E. Witten, Quantum gravity in de Sitter space, hep-th/0106109.

[5] V. Sahni, The cosmological constant problem and Quintessence, astro-ph/0202076.

[6] S. Weinberg, Gravitation and Cosmology(John Wiley and Sons, 1972), pp 375-404.

[7] P.K. Townsend, hep-th/9612121.

[8] A. Strominger, Nucl. PhysB274, 253 (1986); A.S. Majumdar, A. Mukherjee and R.P. Saxena, Mod. Phys .Lett A7, 3647(1992); H.K. Jassal, A. Mukherjee and R.P. Saxena, Int. Jour. Mod. Phy A, Vol 14, No.7 (1999), 1035-1047.

[9] P.K. Townsend, hep-th/0110072 M. Gasperini, F. Piazze and G. Veneziano, grqc/0108016; S. Hellerman, N. Kaloper and L. Susskind, hep-th/0104180.

[10] L. Randall and R. Sundrum, Phys. Rev. Lett83:4690-4693(1999). 